\title{
Suitability of Variance Shift Keying for Real Conditions
}

\author{
Rostislav I. Sokolov and Renat R. Abdullin
}

\begin{abstract}
This paper investigates the possibility of real-life using the brand new type of digital modulation, which implies the transmission of white Gaussian noise whose variance changes in time. That modulation has been entitled as variance shift keying. The signals obtained on its basis have a high level of transmission security, and they even cannot be detected by standard means of matched filtering. However, the paper suggests the decision on how to process these signals at the receiver site. It is proposed to use a specially developed optimal receiver, whose restoration quality appears to be better in comparison with the conventional cumulative receiver. The digital simulation of propagation in frequency selective media has shown the signal with variance shift keying is possible to be used in real conditions since its distribution of instantaneous amplitudes remains Gaussian.
\end{abstract}

Keywords - cumulative receiver, free space propagation, inconstant variance, matched filter, optimal receiver, signal hiding, signal interception, variance shift keying, white Gaussian noise.

\section{INTRODUCTION}

$\mathrm{I}_{\mathrm{s}}^{\mathrm{s}}$ MODERN communication systems, NSS (noiseshaped signals) are used to significantly spread the transmitted signal spectrum in order to provide the great number of subscribers with a communication. The NSS have a row of advantages, the main of which is a high immunity to both broadband and narrowband interference. Apart from that, NSS-based systems have a high energetic concealment and, consequently, a high confidentiality of data transmission. Therefore, the difficult task is not only to recover the data from the NSS without a priori information about the pseudo-random sequence, but also to detect this broadband signal at all [1].

For example, the use of circuit multiplication (broadband transmission based on NSS) in the CDMA (code division

Paper received April 20, 2018; revised August 24, 2018; accepted August 29, 2018. Date of publication December 25, 2018. The associate editor coordinating the review of this manuscript and approving it for publication was Prof. Branimir Reljin.

This paper is a revised and expanded version of the paper presented at the 25th Telecommunications Forum TELFOR 2017 [4].

The work was supported by Act 211 Government of the Russian Federation, contract № 02.A03.21.0006.

The research was executed by the grant of the Ministry of education and science of the Russian Federation (project № 8.2538.2017/4.6).

Rostislav I. Sokolov, and Renat R. Abdullin are with the Engineering School of Information Technologies, Telecommunications and Control Systems, Ural Federal University named after the first President of Russia B.N.Yeltsin, Mira street 19 , 620002 Yekaterinburg, Russian Federation (phone: +7 (343) 3754886; e-mail: r.r.abdullin@urfu.ru) multiple access) systems allows recognizing the signals at the negative values of SNR (signal-to-noise ratio) measured in $\mathrm{dBs}$, ensures the invariance under the multipath propagation and provides all subscribers with a simultaneous operation in a common frequency band [2], [3].

On the other hand, noise-shaped signals also have some disadvantages generally consisting of technical complexity of NSS-based systems implementation. First of all, it is quite difficult to create the large NSS systems with minimal cross-correlation functions. Secondly, it requires using the complex and expensive processing devices, in particular, filters matched with NSS [1], [3].

Additionally, a mandatory requirement for the system operation is the knowledge of coding pseudo-random sequence for signal reconstruction at the receiver site. The knowledge of this sequence makes it possible to intercept and restore the data hidden.

Therefore, this study is aimed to create the NSS-based communication system which does not require receiver to know the pseudo-random sequence. At the same time, the signal transmitted should not be detected by means of conventional spectral analyzers. One variant of systems mentioned above is a system based on a brand new type of digital modulation which we suggested in [4] and entitled as VSK (variance shift keying).

Like any other shift keying, VSK implies the signal parameters change abruptly depending on the bits of original message at specific time intervals. However, the fundamental difference lies in the fact that we propose to transmit only WGN (white Gaussian noise) instead of a conventional harmonic signal traditionally modulated with amplitude, frequency or phase. Obviously, it is quite difficult to find the values of specified parameters for WGN, but WGN has its own parameters, like mean and variance. We have chosen the latter alternative for digital modulation since it is a more viable option because technically the variance is equivalent to the power of signal transmitted.

The main task of present work is to define whether it is even possible to restore VSK signal and how to do it most effectively if so. On the other hand, it is also necessary to show that signal considered is conceptually able to exist for a long time. We need to understand that VSK signal does not go to the single spectral components due to its ultrawide frequency band after a few-meter propagation in free space or frequency-selective media such as real guided lines.

For this reason it is necessary to build a special computer model, which describes VSK-signal propagating in medium 
with frequency-selective fading, and then consider it in order to make sure that despite the signal distortion in frequency spectrum, it is still possible to restore it in time domain.

The results of proposed study are to show the possible application for signal developed.

\section{Mechanism of VARIANCE MOdUlation}

At the first stage of study we considered a unipolar binary NRZ (non-return to zero) pulse sequence as an information signal modulating white Gaussian noise. The very modulation, in this case, is performed by a simple multiplication of the considered sequence and pseudorandom white Gaussian noise with a certain variance value. In these conditions, zero signal values (there are no pulses in initial sequence) can be modulated by additive WGN with another variance, or can remain without modulation.

Obviously, the first option offers more scope for transmission security, since the signal, in this situation, scarcely visually differs from noise. But on the other hand, it also leads to the complication of VSK signal reception, especially under noise conditions, not only for interceptor, but also for subscribers. It is certain that the use of closely spaced variance value is very prospective for a high-speed data transmission system, when modulating sequence has few levels. However, now it looks like a matter for the future. So we have decided to start easily and consider only two-level keying, when the high level of modulating pulse sequence corresponds to the noise with a certain variance, and the second level means an absence of signal at the transmitter output (variance is equal to zero).

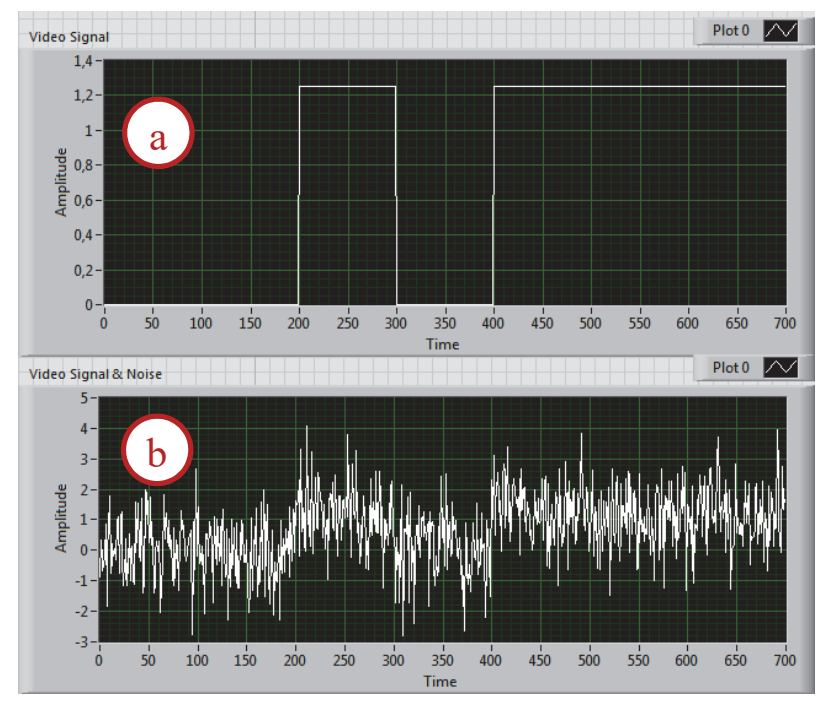

Fig. 1. Mechanism of conventional signal transmission:

(a) initial signal in the form of unipolar NRZ sequence;

(b) mixture of initial signal with $\mathrm{WGN}$ at $\mathrm{SNR}=0$.

Fig. 1 shows the transmitted unipolar binary NRZ sequence and corresponding mixture with the noise at the receiver input. Fig. 2 shows a random signal whose variance changes according to the sequence given in Fig. $1 a$ and the mixture of this signal with noise at the receiver input. The difference in appearance between two specified cases is illdisguised. In the first situation (NRZ signal), despite the high level of noise, the signal received is seen not to be natural, whereas the VSK signal mixed with additive WGN does not visually stand out from natural interference.

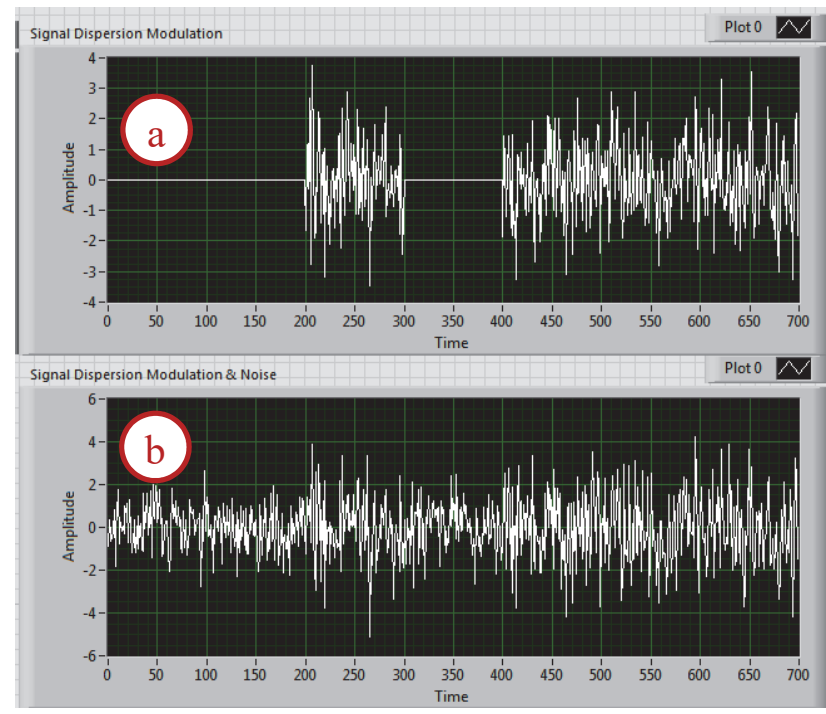

Fig. 2. Mechanism of VSK signal transmission:

(a) the random process whose variance is modulated by initial signal; (b) the mixture of modulated signal with white Gaussian noise at SNR $=0$.

\section{RECEPTION OF SignAls With VARIANCE SHIFT KEYING}

Generally, the operation of standard data transmission systems is simply detected by means of spectral measurements due to the presence of characteristic pronounced spectral harmonics, whose level can be much (ten, hundred or more times) higher than the general level of noise. At the same time the VSK signal really is the noise. That is why its spectrum (Fig. 3a) completely coincides with the spectrum of WGN (Fig. 3b), and VSK signal is impossible to be detected in the mixture with additive noise [5]. Therefore, the use of VSK signal does not make it impossible to restore the signal intercepted as much as it hides the mere fact of transmission.

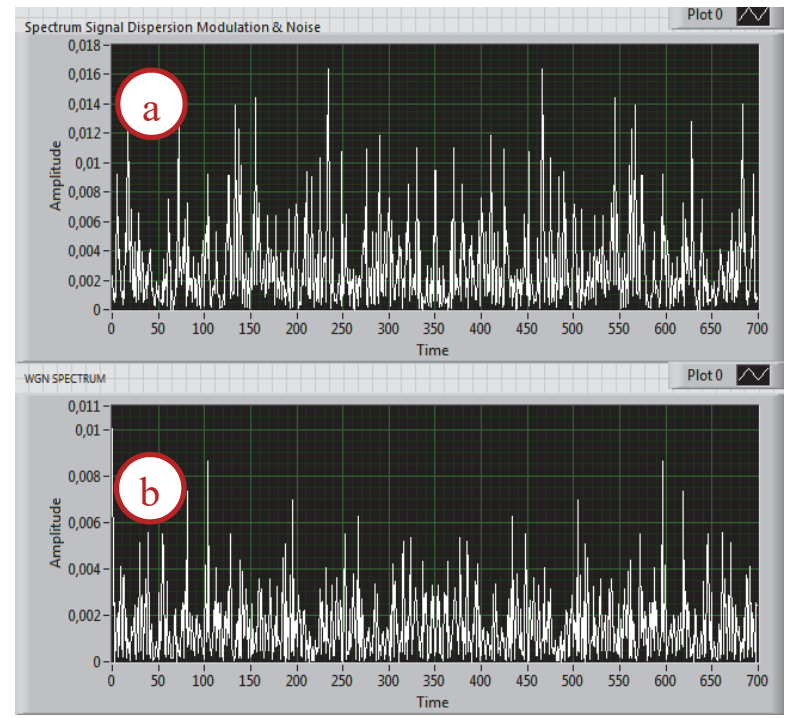

Fig. 3. The spectra of (a) random process with variance shift keying; (b) white Gaussian noise. 


\section{A. VSK Signal Processing with Cumulative Receiver}

The use of correlation analysis and matched filtering methods does not allow detecting the signal at values of SNR less than $0 \mathrm{~dB}$. So, Fig. $4 a$ shows the CCF (cross correlations function) between the signal and noise mixture and reference signal. In this case, the sequence of transmitted pulses is completely detected. Fig. $4 b$ demonstrates the CCF between reference signal and VSK signal mixed with noise. In these conditions, the obtained results do not let reliably identify any transmitted pulse.

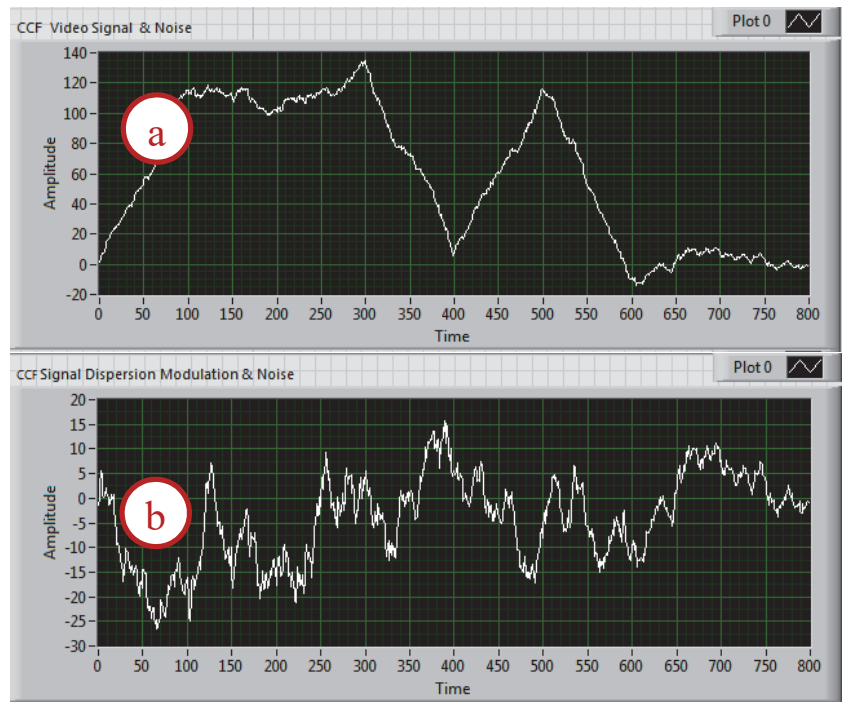

Fig.. 4. Cross-correlation function between the reference signal and mixed with noise (a) conventional unipolar binary NRZ sequence; (b) VSK signal.

Nevertheless, the sections of statistical radio engineering suggest some solutions of the problem. According to them, the VSK signal is possible to be restored by means of a wellknown cumulative receiver [6]. During the study we have implemented the simplest receiver of this type and investigated the results of its operation.

The receiver output produces the sawtooth pulses sequence (Fig. 5). That sequence then goes to the decisionmaking unit, which detects the pulse presence, if the sawtooth pulse exceeds the threshold calculated as follows

$$
L=A V G\left\{\sum_{i=0}^{m-1} y_{i}, \sum_{i=m}^{2 m-1} y_{i}, \ldots, \sum_{i=(k-1) m}^{k m-1} y_{i}\right\},
$$

where $y_{i}$ are the samples of input signal and noise mixture; $m$ is a number of samples per pulse; $k$ is a total quantity of pulses in signal; $A V G$ is a function calculating the arithmetical mean.

We have put two signals mixed with an external noise through the cumulative receiver. The first signal was the conventional unipolar NRZ sequence. And the second one was the newly developed signal with variance shift keying. As the study result we have obtained the dependencies of single pulse correct detection error probability vs SNR. As it is seen from Fig. 6, using the developed type of digital modulation leads to the losses in restoration quality by 8-10 dB in comparison with a conventional NRZ sequence at the values of SNR less than $5 \mathrm{~dB}$.

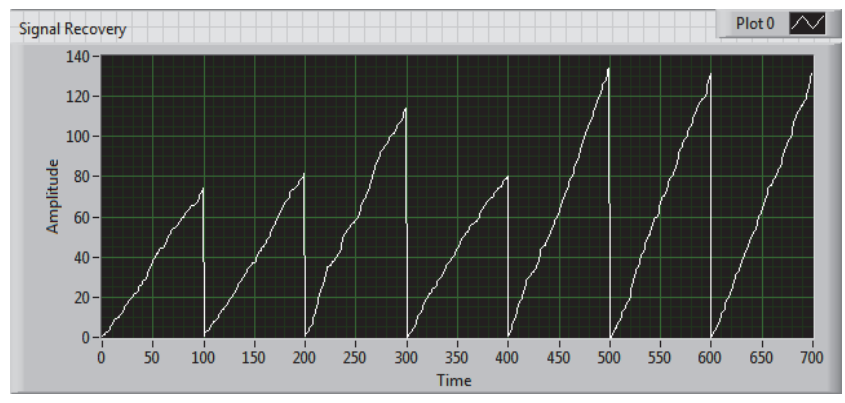

Fig. 5. The sawtooth pulse sequence at the cumulative receiver output.

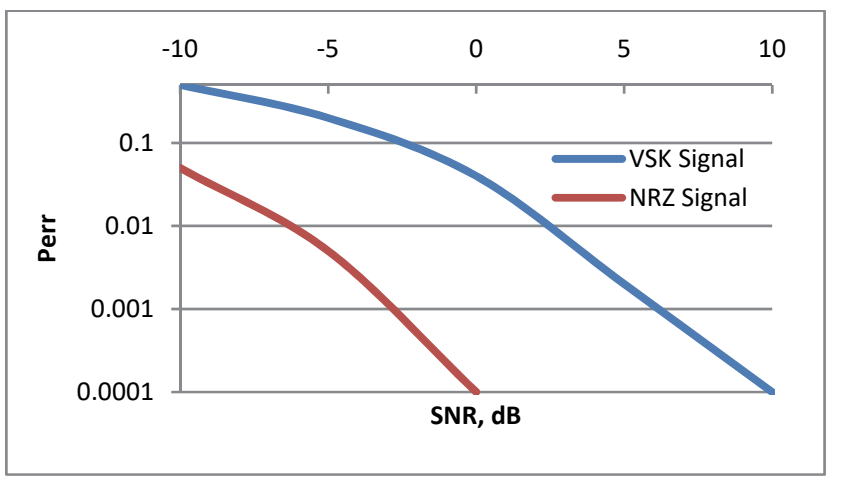

Fig. 6. Dependencies of single pulse correct detection error probability vs signal to noise ratio.

\section{B. VSK Signal Processing with Optimal Receiver}

The other method for VSK signal processing with a better quality is the simulation of the likelihood ratio. In contrast to the optimal receivers based on the average risk minimum criterion we developed in [7] and [8] for the determinateform signals, a priori data, in this case, are the modulating noise variance and the single pulse duration, but not parameters of pulses amplitude and their carrier frequency.

We understand by the term of likelihood ratio the ratio between the distribution density of VSK pulses mixed with external noise and the distribution density of just a noise.

Suppose the process $y(t)$ is observed at the receiver input in discrete moments of time $t_{i}$ :

$$
y_{i}=\left\{\begin{array}{l}
D_{i} n_{i}+z_{i}, \quad \text { if } \Theta=1, \\
z_{i}, \quad \text { if } \Theta=0,
\end{array} \quad i=1,2, \ldots, m,\right.
$$

where $m$ is a total number of observation time samples; $\Theta$ indicates the presence or absence of useful signal modulating the variance $D_{i}$ of white Gaussian noise $n_{i}$.

Gaussian Markov noise $z_{i}$ in (2) describes the external noise given by initial and conditional probability distribution densities:

$$
\begin{gathered}
w_{0}(z)=\frac{1}{\sigma_{z} \sqrt{2 \pi}} \exp \left\{-\frac{z_{0}^{2}}{2 \sigma_{z}^{2}}\right\}, \\
w\left(z_{i+1} / z_{i}\right)=\frac{1}{\sigma_{z} 2 \pi \sqrt{\left(1-R^{2}\right)}} \exp \left\{-\frac{\left(z_{i+1}-z_{i} R\right)^{2}}{2 \sigma_{z}^{2}\left(1-R^{2}\right)}\right\},
\end{gathered}
$$

where $\sigma_{z}$ is a standard deviation of random value $z_{i} ; R$ is a noise correlation coefficient varying from 0 to 1 . On the other hand, the noise $z_{i}$ can be expressed in terms of (2): 


$$
z_{i}=\left\{\begin{array}{l}
y_{i}-D_{i} n_{i}^{*}, \quad \text { if } \Theta=1, \quad i=1,2, \ldots, m . \\
y_{i}, \quad \text { if } \Theta=0,
\end{array}\right.
$$

The noise $n_{i}$ from (2) we have replaced in (5) with one of its realizations $n_{i}^{*}$, since the received signal realization is $a$ priori unknown. After an integration of (5) to (3) and (4) an expression for likelihood ratio $\Lambda_{m}$ looks as follows

$$
\Lambda_{m}=\frac{w_{0}\left(y_{0}-D_{0} n_{0}^{*}\right) \prod_{i=1}^{m} w\left(\left(y_{i+1}-D_{i+1} n_{i+1}^{*}\right) /\left(y_{i}-D_{i} n_{i}^{*}\right)\right)}{w_{0}\left(y_{0}\right) \prod_{i=1}^{m} w\left(y_{i+1} / y_{i}\right)} .
$$

Some mathematical transforms make it possible to obtain an expression for the optimal reception algorithm, tuned to the VSK signal:

$$
\begin{gathered}
l_{m}=\ln \Lambda_{m}=\sum_{i=0}^{m} \frac{\left(y_{i+1}-y_{i} R\right)^{2}}{2 \sigma_{z}^{2}\left(1-R^{2}\right)}- \\
-\sum_{i=0}^{m} \frac{\left(\left(y_{i+1}-D_{i+1} n_{i+1}^{*}\right)-\left(y_{i}-D_{i} n_{i}^{*}\right) R\right)^{2}}{2 \sigma_{z}^{2}\left(1-R^{2}\right)} .
\end{gathered}
$$

Therefore, (7) determines the receiver scheme, which is presented in Fig. 7. The mixture of signal and noise from the receiver input is processed in two parallel channels followed by the adder. In the ideal case, when the first pulse time arrival is precisely known, the result signal goes to the decision-making device, which indicates the presence of single pulse in the input mixture when crossing the threshold $l_{m}$, and its absence otherwise.

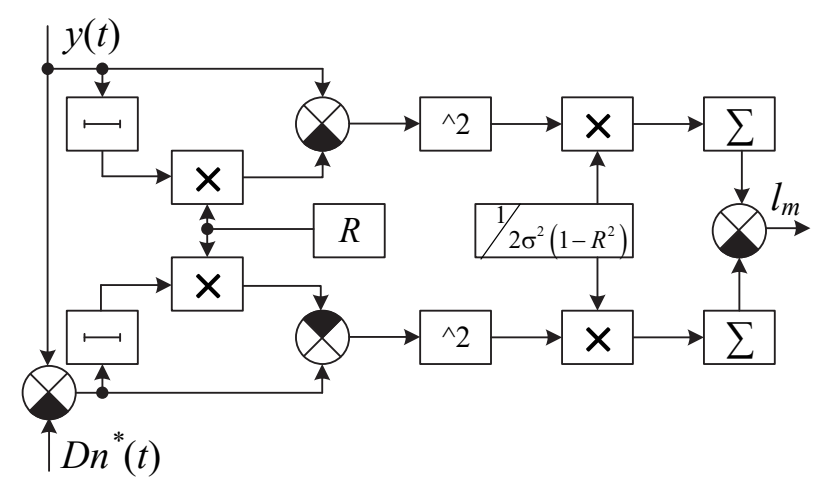

Fig. 7. The block diagram of optimal receiver.

It is worth noting that the ideal case mentioned above is only possible with the use of a precise sync system. In order to organize the synchronization we propose to use parallel processing of signal obtained and its copies, shifted in time by the sample with respect to the previous one in the range of half number of samples per pulse. Opposite to the sync system we developed for radars in [8], from the output of every simple processor the restored sequence goes not to the correlator, but to the unit calculating the difference between the maximal and minimal accumulated peaks of single pulses. Then the maximal value of differences mentioned is found. Therefore, the corresponding sample shift provides the best quality of recovery and synchronization. The scheme of sync system described is presented in Fig. 8.

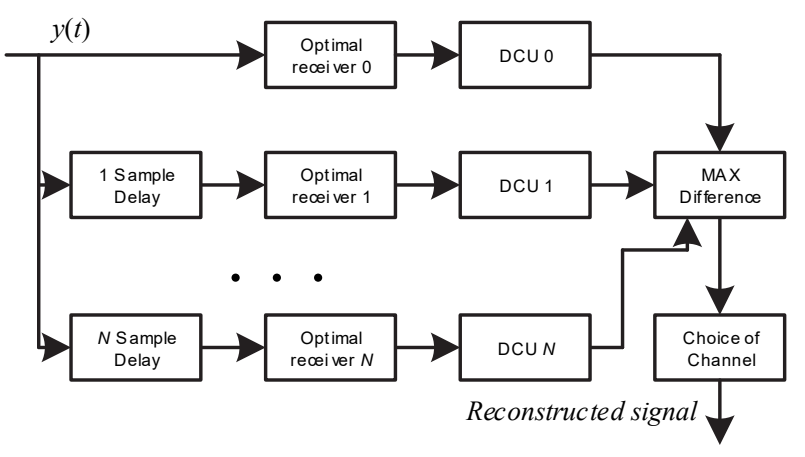

Fig. 8. The block diagram of sync system.

Fig. 9 shows the results of VSK signal restoration by means of the simple cumulative receiver and the newly developed optimal receiver based on the simulation of likelihood ratio. The results of digital simulation have shown the gain of developed receiver in the single pulse restoration quality is up to $2 \mathrm{~dB}$ in comparison with the cumulative receiver when the SNR exceeds $-10 \mathrm{~dB}$.

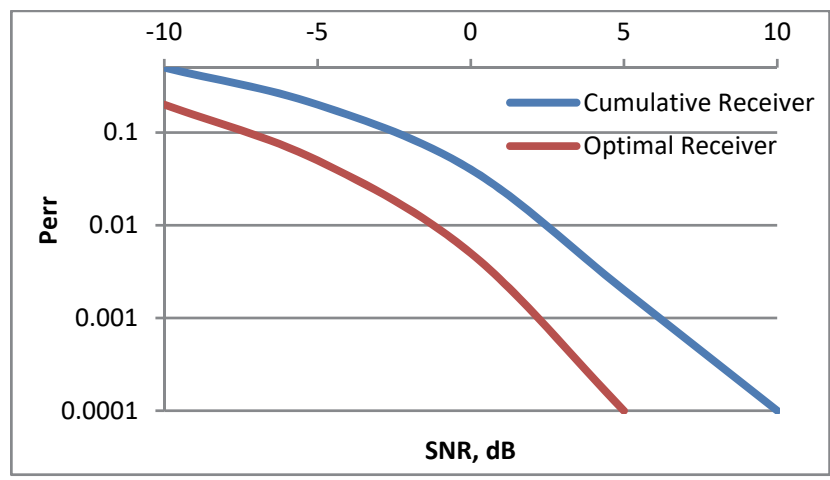

Fig. 9. Comparison of VSK signal restoration quality by the optimal and cumulative receivers.

\section{VSK SIgNAL PROPAGATION}

Despite all their advantages VSK signals call for additional investigations. The fact is that the signal propagating in terrain environment or other media with frequency-selective fading has a different attenuation in different frequency bands. And since the proposed signal is ultra wideband, a dispersion can lead to the signal nonlinear distortion and, consequently, to the change in distribution law of instantaneous amplitude.

In order to establish the practical application limits of propagating VSK-signals, we have conducted the computer simulation, which helped us to estimate the restoration quality for the signals nonlinearly distorted in time domain. For this purpose we built the digital model, which firstly made it possible to expand the VSK signal in spectral components via a fast Fourier transform. After that, every spectral component was multiplied by its own coefficient, and then an inverse fast Fourier transform was made.

In present work we focused on propagation in free space, when the electromagnetic field intensity at the receiver site decreases proportionally to $\lambda / 4 \pi d$, where $\lambda$ is a wavelength corresponding to every separate spectral component, $d$ is a distance from transmitter. So, the coefficient we used for 
multiplication was $\lambda / 4 \pi d$. The results of transforms are presented in Fig. 10.

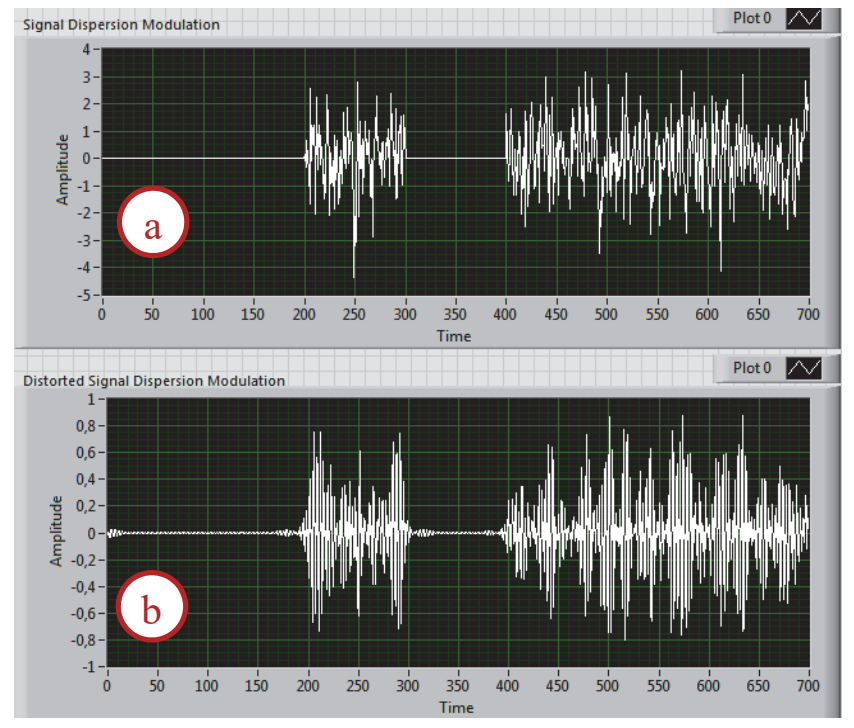

Fig. 10. The VSK signal: (a) transmitted,

(b) received 10 meters away.

In addition to the fact that the very VSK signal gets distorted during the propagation, it is additionally mixed with external noise, which basically provides the transmission security. Therefore, an observation of SNR values close to $0 \mathrm{~dB}$ at the receiver input is the common occurrence. Fig. 11 shows an appearance of VSK signal distorted by propagation and mixed with noise, when SNR at the receiver input is equal to $0 \mathrm{~dB}$.

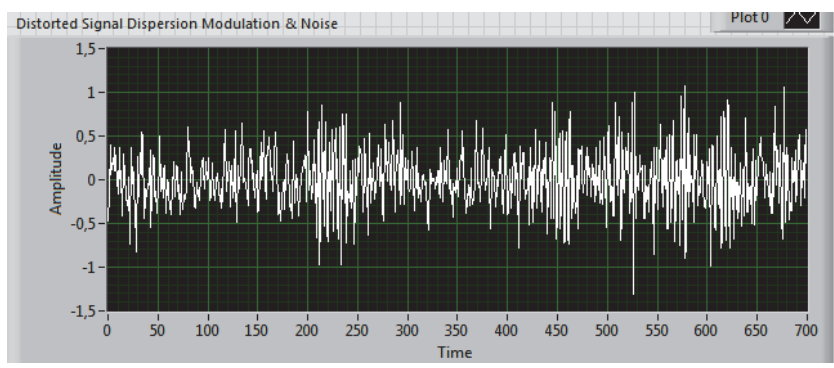

Fig. 11. The mixture of distorted VSK signal and noise, when SNR at the receiver input is equal to $0 \mathrm{~dB}$.

During the digital experiment the mixture of distorted VSK signal and external noise has also been processed with the developed optimal receiver. The results presented in Fig. 12 show that the optimal receiver does not have significant losses in restoration quality for VSK signal (with band of $10 \mathrm{GHz}$ ) distorted by fading in 10-meter distance in comparison with undistorted signal, when SNR at the receiver input is equal to $0 \mathrm{~dB}$.

We have also conducted the experiment for other values of distance $d$ and SNR. The optimal receiver demonstrates acceptable results when signal propagating up to $100 \mathrm{~m}$ at SNR values higher than $0 \mathrm{~dB}$ (Fig. 13).

Therefore, it is possible to conclude that the VSK signal propagating through the space retains the normal distribution of its instantaneous amplitude. It is highly likely that the attachment of propagation factor to the model developed will deliver the same results.

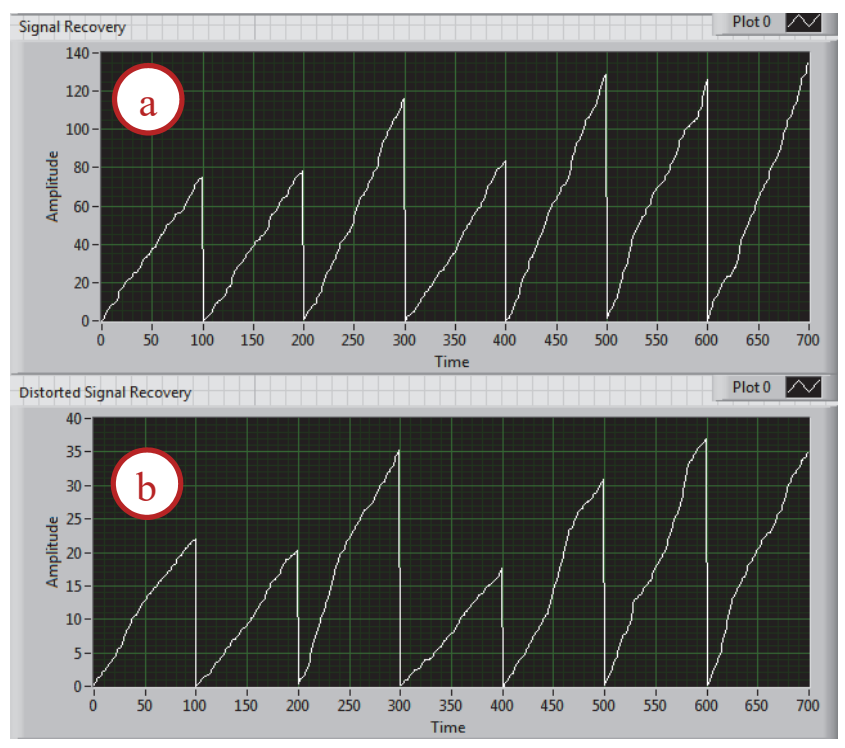

Fig. 12. The results of restoration for (a) undistorted and (b) distorted VSK signals. SNR $=0 \mathrm{~dB}$.

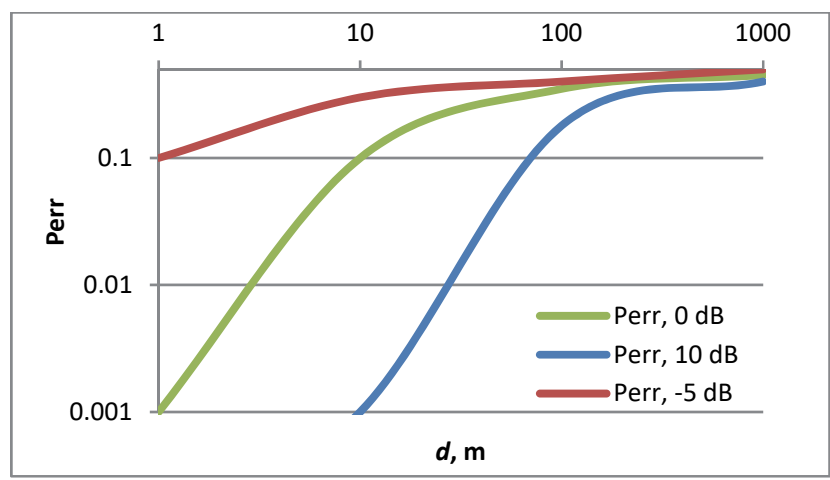

Fig. 13. The dependence of VSK signal restoration quality on distance of its propagation.

\section{DISCUSSION}

Of course, these days the VSK signal is still a novelty and it seemed it could be effective only because not many people are aware of it. However, this is not the case. Even if an attacker does expect that VSK signal can be used in transmission, he unlikely will be able to intercept it.

The fact is that the use of optimal receiver based on average risk minimum criterion strictly requires a priory information on system parameters, which include the single pulse duration, sampling rate and arrival time of the first pulse.

At the same time, almost all modern wideband detection receivers operate in the frequency domain, previously analyzing the spectral components in the air. After detecting the distinctive spectral components, the receiver either performs operations of spectrum transform to a time signal with loss of the phase component or starts processing the input signal in time-domain with the corresponding parameters of center frequency and bandwidth.

However, since the given values of SNR do not allow the wideband receivers to extract distinctive (different from noise) spectral components, it is impossible to restore the VSK signal by none of the above mentioned methods. 
We do not know of any detection receiver able to perform parallel or quasi-parallel processing at every possible frequency with various bandwidths and acceptable quality. Moreover, at the current stage of microprocessor technology development the creation of similar system looks incredible.

Therefore, the interception and following restoration of messages modulated with VSK at specified values of SNR without a priori information about the system is an almost unachievable task, even using the special algorithms of optimal signal processing.

\section{CONCLUSION}

The model of signal with a brand new type of modulation entitled as variance shift keying has been developed. This modulation is based on transmission of a random signal whose variance is changing in time. The proposed system was found to have an extra security of signal transmission both in time and frequency domain, which can be quite useful in telephone-communication and telegraph systems.

The phase-locked optimal receiver based on simulation of likelihood ratio has been developed for VSK signals. That receiver has a gain of $2 \mathrm{~dB}$ in restoration quality in comparison with a conventional cumulative receiver, when SNR exceeds the value of $-10 \mathrm{~dB}$.

In order to establish the practical limits of VSK signal application in terrain environment with dispersion properties, the digital simulation has been undertaken. The digital experiment has shown that a VSK signal is possible to be transmitted and, moreover, recovered in real conditions by means of a specially developed optimal receiver.

\section{REFERENCES}

[1] V. V. Dubrovskiy. (2003, June). CDMA - sight of professional [CDMA - vzglyad professionala]. Radioradar. Available: http://www.radioradar.net/articles/scientific_technical/cdma.html

[2] J. G. Proakis, Digital Communications. New York: McGraw-Hill Education, 2007, p. 1150.

[3] J. S. Lee, L. E. Miller, CDMA System Engineering Handbook, Artech House Publishers, 1998, p. 1228.

[4] R. I. Sokolov, R. R. Abdullin, "Variance Shift Keying in Wideband Communication Systems," in Proc. 25th Telecommunication Forum (TELFOR), Belgrade, Serbia, 2017, pp. 386-388.

[5] L. S. Gutkin. The theory of optimal receiving methods with fluctuation interference [Teoriya optimalnykh metodov radiopriyema pri fluktuatsionnikh pomekhah]. 2nd ed. Moscow: Sov. Radio, 1972, p. 448.

[6] V. I. Tikhonov. Statistical radiotechnics [Statisticheskaya radiotekhnika]. Moskow: Sov. Radio, 1966.

[7] R. I. Sokolov, R. R. Abdullin, D. A. Dolmatov, "Development of Synchronization System for Signal Reception and Recovery from Usb-Keyboard Compromising Emanations," in Proc. 2016 2nd International Conference on Industrial Engineering, Applications and Manufacturing (ICIEAM 2016), Chelyabinsk, Russia, 2016.

[8] D. A. Dolmatov, R. R. Abdullin, R. I. Sokolov, "Phase-Locked Radar Impulse Optimal Receiver by Average Risk Minimum Criterion," in Proc. 2017 IEEE Radio and Antenna Days of the Indian Ocean (RADIO), Cape Town, South Africa, 2017. 